\title{
riccafd
}

Revista Iberoamericana de Ciencias de la Actividad Física y el Deporte

\section{INFLUENCIA DE LA DIETA Y HÁBITOS \\ FÍSICO-SALUDABLES EN ESCOLARES}

\section{INFLUENCE OF DIETARY AND PHYSICAL HABITS IN SCHOOLCHILDREN}

Ubago-Jiménez, J.L.. ${ }^{1 F C}$; Chacón-Cuberos, R. ${ }^{2 B D}$; Puertas-Molero, P. ${ }^{3 \mathrm{BC}}$; Ramírez-Granizo, I.A. ${ }^{4 A C}$

${ }^{1}$ Departamento de Didáctica de la Expresión Musical, Plástica y Corporal, Universidad de Granada, España, jlubago@ugr.es

2 Departamento de Métodos de Investigación y Diagnóstico en Educación, de Granada, España, rchacon@ugr.es

${ }^{3}$ Departamento de Didáctica de la Expresión Musical, Plástica y Corporal, de Granada, España, pilarpuertas@correo.ugr.es

${ }^{4}$ Departamento de Didáctica de la Expresión Musical, Plástica y Corporal, Universidad de Granada, España, irwinrg@ugr.es

Responsabilidades

A Diseño de la investigación

${ }^{B}$ Recolector de datos

${ }^{\mathrm{C}}$ Redactor del trabajo

D Tratamiento estadístico

E Apoyo económico

F Idea original y coordinador de toda la investigación

Recibido el 5 de enero de 2020

Aceptado el 26 de febrero de 2020

Correspondencia: Irwin A. Ramírez-Granizo. irwinrg@ugr.es

DOI: http://dx.doi.org/10.24310/riccafd.2020.v9i1.8306

\section{RESUMEN}

La obesidad se ha convertido en una de las pandemias del s XXI y más concretamente entre la población escolar. Así pues, el presente trabajo tiene como objetivo el de describir los niveles de obesidad, el nivel de la dieta y la práctica de actividad física en alumnos de primaria. Para ello la muestra está compuesta por 150 estudiantes de educación primaria de tres centros públicos de Granada de entre 10-12 años (M=10.9; D.T.=1.8). Se ha utilizado el cuestionario KIDMED que ha dado como principales resultados que la muestra seleccionada se centra en torno a la dieta mejorable y óptima. Como principales conclusiones se extrae que la muestra presenta una dieta óptima y que, los niños practican deporte 
colectivo con contacto en mayor porcentaje que las niñas, mientras que estas prefieren los deportes individuales sin contacto.

Palabras clave: dieta mediterránea, obesidad, escolares.

\section{ABSTRACT}

Obesity has become one of the pandemics of the 21st century and more specifically among the school population. Therefore, this work aims to describe the levels of obesity, the level of diet and the practice of physical activity in primary school students. To this end, the sample is made up of 150 primary school students from three public schools in Granada aged 10-12 (M=10.9; S.D.=1.8). The KIDMED questionnaire was used, the main results of which were that the selected sample focuses on the best and best diet. The main conclusions are that the sample presents an optimal diet and that boys practice collective sports with contact in a higher percentage than girls, while girls prefer individual sports without contact.

Key words: mediterranean diet, obesity, schoolchildren.

\section{INTRODUCCIÓN}

Los problemas en cuanto a la obesidad constituyen una de las pandemias más extendidas a lo largo y ancho del globo terráqueo en el siglo XXI, [1]. Entre las principales causas que son detonante de esta enfermedad se encuentran los escasos hábitos físico-saludables, que se ven acentuados por el aumento del sedentarismo o una dieta inapropiada $[2,3]$.

No se puede pasar por alto al hablar de obesidad, la modalidad de dieta seguida. En nuestro contexto, aunque cada vez es más globalizado, el predominio lo ostenta la dieta mediterránea (DM), la cual es una de las más saludables [4-6]. En general, es una dieta de bajo contenido de alimentos procesados y, por lo tanto, una dieta baja en grasas y azúcares refinados, o lo que es lo mismo, una dieta alta legumbres, cereales, frutas y verduras.

La práctica deportiva, además de ser beneficiosa para la capacidad cardiopulmonar y mejora del tono muscular, se asocia con la propia percepción que tiene la persona de sí misma. Estos beneficios generan un incremento del autoconcepto y la propia autoestima. Además, también promueve situaciones de trabajo en equipo, facilitando las relaciones intersociales, así como mejorar el rendimiento académico [7].

A pesar de ello, en las últimas décadas se aprecia una importante reducción de la práctica de actividad física, especialmente en el periodo crítico que comprende la temprana adolescencia [8,9]. Asimismo, el auge de conductas perjudi- 
ciales para la salud generan importantes inquietudes a nivel nacional e internacional [10].

De forma que la adquisición de hábitos y estilos de vida saludables durante esta etapa, se hacen necesarias para que se instauren en la vida adulta, haciendo hincapié en los dos pilares básicos: la actividad física y la alimentación [11].

La inactividad física y, por ende, el sedentarismo, son otra de las principales causas del mal endémico como es el sobrepeso [1]. Los elevados porcentajes de obesidad ligada a hábitos sedentarios en escolares y a una baja calidad de la dieta, llevan a la realización del presente estudio con los objetivos de describir los niveles de obesidad, el nivel de la dieta y la práctica de actividad física en alumnos de primaria.

Asimismo, el objetivo principal del presente estudio es el conocer la relación existente entre la DM y la práctica de actividad física en una muestra de escolares.

\section{MATERIAL Y MÉTODOS}

\section{Participantes}

Para el estudio se obtuvo una muestra de 150 estudiantes de Educación Primaria de la provincia de Granada, representados en un 48\% ( $N=72)$ por niños y en un $52 \%(\mathrm{~N}=78)$ por niñas, cuyas edades están comprendidas entre 10-12 años $(\mathrm{M}=10.9$; D.T.=1.8).

\section{Instrumentos}

Primeramente, se elaboraron una serie de cuestiones Ad Hoc, en las que se preguntaba por el tipo y práctica de actividad física. Para medir la DM se utilizó el test KIDMED elaborado por Serrá-Majem et al., [12]. Consiste en una batería de 16 ítems con respuesta afirmativa/negativa. Los valores que se obtienen oscilan de -4 a 3 (dieta pobre), entre 4 y 7 (dieta mejorable) y, entre 8 y 12 (dieta óptima).

Además, para conocer el tipo de deporte practicado se hizo un cuestionario ad hoc que integraba las preguntas ¿practicas algún deporte? respondiendo mediante un "si" o un "no" y ¿qué deporte practicas? respondiendo diciendo el deporte practicado. Las respuestas de la segunda pregunta se categorizaron dividiendo los deportes en colectivos e individuales, con y sin contacto.

\section{Procedimiento}

Se elaboró una carta de presentación desde el Departamento de Didáctica de la Expresión Musical, Plástica y Corporal de la Universidad de Granada con el fin de informar a los centros educativos del fin del estudio. Una vez obtenidos los 
permisos de los colegios, se procedió a informar a los tutores legales del alumnado, obteniendo su consentimiento informado. Esta etapa se realizó en horario lectivo, con la presencia de los investigadores con el fin de asegurar la correcta aplicación de los instrumentos. Se han cumplimiento de las normas éticas de la Declaración de Helsinki de 1975 y se ha respetado el derecho de confidencialidad de los participantes.

\section{Análisis de los datos}

Se utilizó el programa estadístico SPSS ${ }^{\circ} 24.0$ para el análisis de los datos para determinar los parámetros estadísticos (frecuencias, medias y tablas de contingencia). Los estadísticos descriptivos del estudio se muestran como media \pm desviación estándar. Las diferencias entre géneros fueron analizadas mediante análisis de varianza simple (ANOVA). La dieta mediterránea, valorada mediante el KIDMED, fue analizada mediante una correlación no paramétrica de Spearman (rho) para el total de la muestra y diferenciada por género. Para analizar las asociaciones entre la actividad física y la dieta mediterránea se utilizó el coeficiente de la correlación de Pearson, estableciendo el nivel de significación en $p<.05$.

\section{RESULTADOS}

Tras analizar los resultados, se obtuvo que únicamente el 9,3\% de los participantes presentan una dieta de baja calidad. Por el contrario, el grueso de la población se encuentra repartido entre la dieta mejorable $(42,2 \%)$ y la dieta óptima

Tabla 1. Relación entre género y dieta mediterránea.

\begin{tabular}{|c|c|c|c|c|c|}
\hline \multicolumn{2}{|c|}{ Sexo } & \multicolumn{4}{|c|}{ Dieta Mediterránea } \\
\hline & & \multirow{2}{*}{$\frac{\text { Baja calidad }}{8}$} & \multirow{2}{*}{$\begin{array}{c}\text { Mejorable } \\
25\end{array}$} & \multirow{2}{*}{$\frac{\text { Óptima }}{39}$} & \multirow[t]{2}{*}{ Sig. } \\
\hline & Recuento & & & & \\
\hline \multirow[t]{3}{*}{ Masculino } & \%Sexo & $10.2 \%$ & $39.8 \%$ & $50 \%$ & - \\
\hline & \%Deporte & $66.7 \%$ & $57.4 \%$ & $62.8 \%$ & \\
\hline & Recuento & 5 & 29 & 29 & \\
\hline \multirow[t]{3}{*}{ Femenino } & \%Sexo & $7.9 \%$ & $46 \%$ & $46 \%$ & - \\
\hline & \%Deporte & $33.3 \%$ & $42.6 \%$ & $37.2 \%$ & \\
\hline & Recuento & 15 & 68 & 78 & \\
\hline \multirow[t]{2}{*}{ Total } & \%Sexo & $9.3 \%$ & $42.2 \%$ & $48.4 \%$ & - \\
\hline & \%Deporte & $100 \%$ & $100 \%$ & $100 \%$ & \\
\hline
\end{tabular}


Ante estos datos, se hizo un análisis relacional de la edad y género con la DM en el que no se encontraron diferencias estadísticamente significativas. En cambio, sí se obtuvieron diferencias estadísticamente significativas en cuanto al género y el tipo de deporte practicado. Se puede observar como las niñas practican deportes del tipo individual sin contacto, sin embargo, el los niños practican deportes de tipo colectivo con contacto $(55,1 \%)$.

Tabla 2. Relación entre el género y el tipo de deporte practicado $(p=, 000)$.

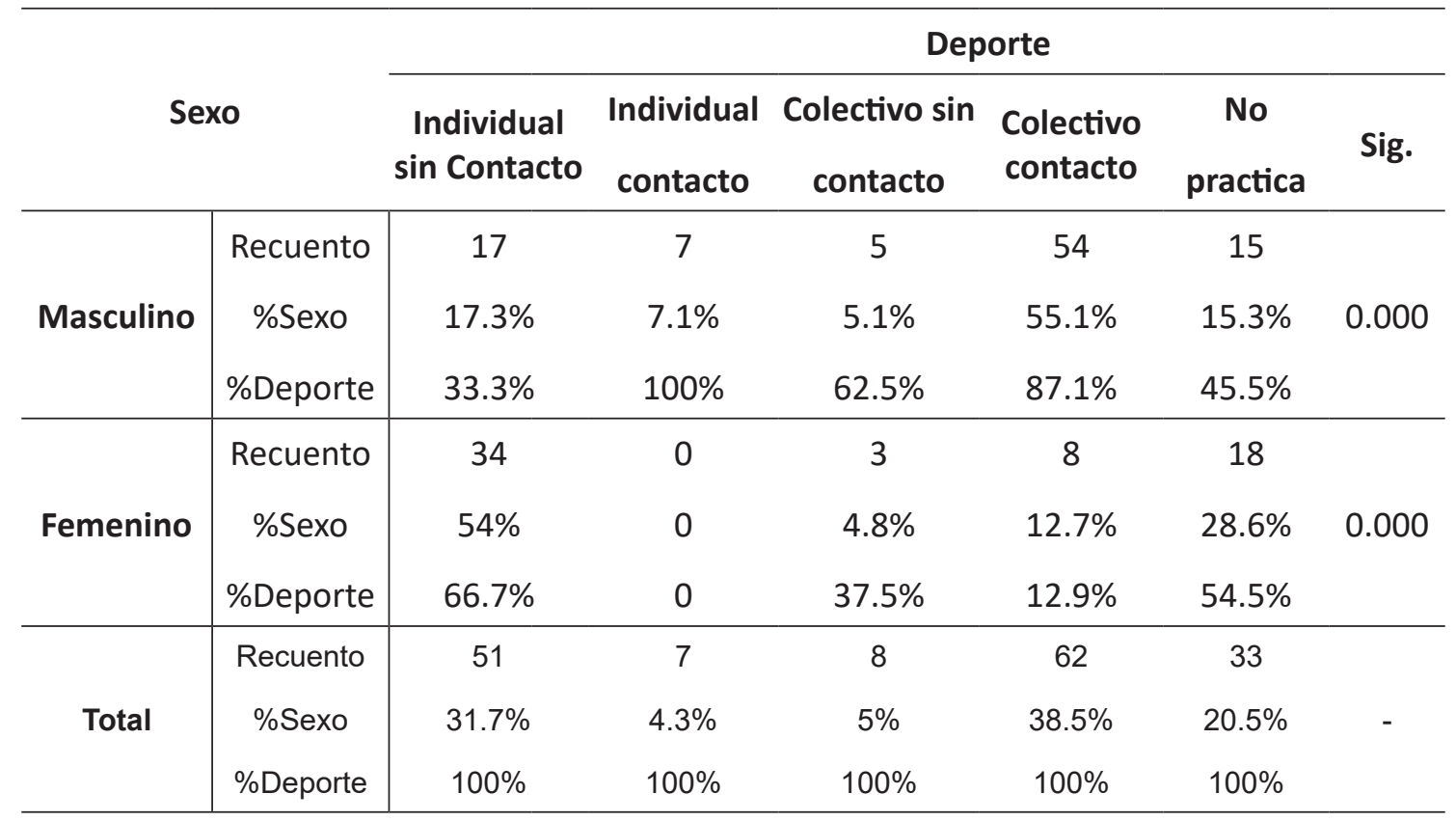

Finalmente se muestran los coeficientes de correlación entre la práctica deportiva y la adherencia a la DM se encuentra (Tabla 3). En relación a la óptima adherencia a la DM encontramos como resultado más significativo una correlación positiva con la práctica deportiva individual con contacto $(r=0.328)$, a la práctica de deporte colectivo con contacto $(r=0.350)$ y la práctica deportiva colectiva sin contacto $(r=0.401)$. Asimismo, la baja calidad de la dieta revela una correlación positiva con los participantes que no practican deporte $(r=0.345)$ y con la práctica deportiva individual sin contacto $(r=0.459)$.

Tabla 3. Correlaciones entre la DM y el tipo de deporte practicado.

\begin{tabular}{cccccc}
\hline & $\begin{array}{c}\text { Individual sin } \\
\text { Contacto }\end{array}$ & $\begin{array}{c}\text { Individual } \\
\text { contacto }\end{array}$ & $\begin{array}{c}\text { Colectivo } \sin \\
\text { contacto }\end{array}$ & $\begin{array}{c}\text { Colectivo } \\
\text { contacto }\end{array}$ & $\begin{array}{c}\text { No } \\
\text { practica }\end{array}$ \\
\hline Baja Calidad & $\mathbf{0 . 4 5 9 * *}$ & 0.047 & 0.121 & 0.027 & $\mathbf{0 . 3 4 5 * *}$ \\
Mejorable & 0.106 & 0.195 & 0.153 & 0.001 & 0.198 \\
Óptima & 0.122 & $\mathbf{0 . 3 2 8 ^ { * * }}$ & $\mathbf{0 . 4 0 1 * *}$ & $\mathbf{0 . 3 5 0 * *}$ & 0.027 \\
\hline
\end{tabular}




\section{DISCUSIÓN}

El propósito del estudio fue analizar las relaciones entre las habilidades físicosaludables y la DM en estudiantes de educación primaria. Al establecerse una relación estadística entre las variables de la edad y género con la DM, la mayor parte de la población estudiada se reparte casi de forma equitativa entre la una dieta mejorable y una óptima, $[13,14]$. Este hecho induce a pensar que la muestra estudiada presenta un patrón de adhesión a la DM aceptable casi en su totalidad, pero no óptimo $[13,15]$. Por el contrario, algunos artículos fundamentan que más del $45 \%$ de los niños españoles padecen sobrepeso u obesidad [15]. Este hecho puede deberse a las características del contexto escolar del centro, así como de las propias peculiaridades familiares de cada uno de sujetos que participaron en la investigación [16].

Al observar la relación entre las variables del género y la adhesión a la dieta mediterránea, se pudo apreciar niveles muy similares entre niños y niñas, presentando 1 de cada 10 una dieta de baja calidad. Dichos datos presentan similitudes con los obtenidos en otros estudias realizados como es el caso de [17], esto puede deberse a la zona geográfica donde se estudió la muestra, cuya proximidad al mediterráneo favorece la adhesión a este tipo de dieta.

Por otra parte, y siguiendo con los datos obtenidos, 5 de cada 10 niñas practican deporte de carácter individual y sin contacto. Por el contrario, la realización de actividad física por parte de los niños se centra en el deporte colectivo con contacto, el cual practican 6 de cada 10 niños, esto puede deberse, como ya se ha comentado anteriormente, a una etapa de mayor actividad física en los escolares. Por otra parte, la práctica de estos tipos de deporte tanto de niños como de niñas puede deberse a la tendencia social, en la cual predominan en el género masculino deportes como el futbol o la gimnasia rítmica en el género femenino.

Del mismo modo y en consonancia con otros estudios realizados por autores como [18,19], quienes afirman en sus investigaciones que el género masculino es más propenso a la realización de actividad física que el femenino, esto se ve confirmado con los datos obtenidos del estudio realizado, en el que el género femenino presenta un nivel de inactividad física o sedentarismo de 3 de cada 10, siendo ligeramente superior al masculino.

Por último, al observar las relaciones establecidas entre la obesidad y la dieta y la obesidad y la actividad física, se puede entender que no se encontraran diferencias significativas, pues como se muestra anteriormente en las correspondientes tablas, los niveles más bajos se detectaron en alumnos con obesidad y con una dieta de mala calidad encontrándose en una proporción de 1 de cada 10. Del mismo modo se observó la relación entre alumnos con normopeso y que realizan algún tipo de práctica deportiva siendo 8 de cada 10, o por el contrario la presencia de sobrepeso u obesidad relacionada con el sedentarismo encontrándose ambas en una proporción de 1 de cada 10. 
Como principales concusiones se extrae como la muestra seleccionada presenta una dieta óptima. También cabe resaltar la participación o realización de los alumnos en algún tipo de actividad física, la práctica de deporte colectivo con contacto predomina ligeramente sobre la práctica de deporte individual sin contacto.

\section{REFERENCIAS BIBLIOGRÁFICAS}

1. Chacón-Cuberos, R., Castro-Sánchez, M., Muros-Molina, J. J., Espejo-Garcés, T., Zurita-Ortega, F., y Linares-Manrique, M. Adhesión a la dieta mediterránea en estudiantes universitarios y su relación con los hábitos de ocio digital. Nutrición Hospitalaria, 2016, 33(2): 405-410.

2. Sánchez-Cruz, J.J., Jiménez-Moleón, J.J., Fernández-Quesada, F., y Sánchez, M.J. Prevalencia de obesidad infantil y juvenil en España en 2012. Revista española de cardiología, 2013, 66(5): 371-376.

3. Martínez-López, E.J. y Redecillas-Peiró, M.T. Prevalencia de sobrepeso y obesidad en escolares de la provincia de Jaén. Revista Internacional de Medicina y Ciencias de la Actividad Física y el Deporte, 2011, 11(43): 472-490.

4. Alonso, F.J., Carranza, M.D., Rueda, J.D., y Naranjo, J. (2014). Composición corporal en escolares de primaria y su relación con el hábito nutricional y la práctica reglada de actividad deportiva. Revista Andaluza de Medicina del Deporte, 2014, 7(4): 137-142.

5. Arriscado, D., Dalmau, J.M., Zabala, M., y Muros, J.J. Adherencia a la dieta mediterránea en escolares: factores influyentes y relación con otros hábitos de vida. En Dieta Mediterránea Avances en Alimentación, Nutrición y Dietética (pp. 67-81). Madrid: Sociedad Española de Dietética y Ciencias de la Alimentación; 2014.

6. Menéndez-Santurio, J. I., y Fernández-Río, J. Hábitos de alimentación y actividad física de Menores Extranjeros No acompañados (MENAs): un estudio de caso. Retos. Nuevas tendencias en Educación Física, Deporte y Recreación, 2015, 28 : 33-39.

7. García-González, A. J. y Froment, F. Beneficios de la actividad física sobre la autoestima y la calidad de vida de personas mayores. Retos, 2018, 33(33): 3-9.

8. Beltrán-Carrillo, V., Sierra, C., Jiménez-Loaisa, A., González-Cutre, D., MartínezGalindo, C., y Cervelló, E. Diferencias según género en el tiempo empleado por adolescentes en actividad sedentaria y actividad física en diferentes segmentos horarios del día. Retos, 2017, 31: 3-7.

9. Revuelta, L., Infante, G., y Axpe, I. Contexto familiar e implicación en el ámbito físicodeportivo durante la adolescencia. International Journal of Developmental and Educational Psychology. Revista INFAD de Psicología, 2017, 6(1): 311-320.

10. Zurita-Ortega, F., Salvador-Pérez, F., Knox, E., Gámiz-Sánchez, V., Chacón-Cuberos, R., Rodríguez-Fernández, S. y Muros, J.J. Physical activity and health-related quality of life in schoolchildren: structural equations analysis. Anales de psicología, 2018, 34(2): 385-390.

11. Chacón-Cuberos, R., Muros-Molina, J.J., Chacón-Zagalaz, J., Zagalaz-Sánchez, M.L., Castro-Sánchez, M. y Zurita-Ortega, F. Actividad física, dieta mediterránea y 
clima motivacional hacia el deporte en escolares de la provincia de Granada: un modelo de ecuaciones estructurales. Nutrición hospitalaria, 2018, 35(4): 774-781.

12. Serrá-Majem, L., Ribas, L., Ngo, J., Ortega, R.M., García, A., Pérez-Rodrigo, C., et al. Food, youth and the Mediterranean diet in Spain. Development of KIDMED, Mediterranean diet quality index in children and adolescents. Public Health Nutrition, 2004, 7(7): 931-935.

13. Muntaner-Mas, A., Vidal-Conti, J., Cantallops, J. Borrás, P., y Palou, P. Obesity and physical activity patterns among Balearic Islands children and adolescents: a crosssectional study. Journal of Human Sport and Exercise, 2017, 12(2): 333-348.

14. Espejo-Garcés, T., Cabrera-Fernández, Á., Castro-Sánchez, M., López-Fernández, J.F., Zurita-Ortega, F., y Chacón-Cuberos, R. Modificaciones de la obesidad a través de la implementación de herramientas físico-posturales en escolares. Retos. Nuevas Tendencias en Educación Física, Deporte y Recreación, 2015, 28: 78-83.

15. Pinel, C., Chacón, R., Castro, M., Espejo, T., Zurita, F., y Pérez, A. Diferencias de género en relación con el Índice de Masa Corporal, calidad de la dieta y actividades sedentarias en niños de 10 a 12 años. Retos, Nuevas tendencias en Educación Física, Deporte y Recreación, 2017, 31: 176-180.

16. Morales Falo, E.M., Sánchez Moreno, C., Esteban, A., Alburquerque, J.J., y Garaulet, $\mathrm{M}$. Calidad de la dieta «antes y durante» un tratamiento de pérdida de peso basado en dieta mediterránea, terapia conductual y educación nutricional. Nutrición Hospitalaria, 2013, 28(4): 980-987.

17. Mauro-Martins, J., Honório, S., Costa, A., Batista, M., y Cardoso, J. Longitudinal study of physical fitness levels, BMI and Childhood obesity in school context. Journal of Human Sport and Exercise, 2014, 9(2): 645-655.

18. San Román-Mata, S. Mediterranean diet and physical activity in young people and adults of Melilla. ESHPA - Education, Sport, Health and Physical Activity, 2019, 3(2): 209-222.

19. García-González, F., Ferrer-García, J.C., Pablos-Monzó, C., Elvira-Macagno, L., Martín-Rodríguez, M., Albalat-Galera, R., Pablos-Abella, C. Beneficios de un programa de ejercicio físico en mujeres obesas postmenopáusicas. RICYDE. Revista internacional de ciencias del deporte, 2014, 38(10): 346-365. 